Draft VERSION OCTOBER 24, 2018

Preprint typeset using LATEX $_{\mathrm{E}}$ style emulateapj v. 4/12/04

\title{
A PANCHROMATIC VIEW OF PKS 0558-504: AN IDEAL LABORATORY TO STUDY THE DISK-JET LINK
}

\author{
M. Gliozzi
}

George Mason University, 4400 University Drive, Fairfax, VA 22030

\section{I.E. PAPADAKIS}

Physics Department, University of Crete, 71003 Heraklion, Crete, Greece and Foundation for Research and Technology - Hellas, IESL, Voutes, 71110 Heraklion, Crete, Greece

D. GRUPE

Department of Astronomy and Astrophysics, Pennsylvania State University, 525 Davey Lab, University Park, PA 16802, USA

W.P. Brinkmann, C. Raeth

Max-Planck-Institut für extraterrestrische Physik, Postfach 1312, D-85741 Garching, Germany

\author{
L. KEDZiORA-CHUdCZER \\ Australia Telescope National Facility, CSIRO, P.O. Box 76, Epping, NSW 1710 Currently at the School of Physics, UNSW, Sydney, \\ NSW 2052 Australia \\ Draft version October 24, 2018
}

\begin{abstract}
PKS 0558-504 is the brightest radio-loud Narrow-Line Seyfert 1 galaxy at X-ray energies. Here we present results from the radio, optical, UV, and X-ray bands obtained with SWIFT, XMM-Newton, and $A T C A$ during a 10-day monitoring campaign in September 2008. The simultaneous coverage at several wavelengths makes it possible to investigate in detail the broadband spectral energy distribution (SED) and the energetic of this source. The main results can be summarized as follows. The ATCA reveals the presence of an extended radio emission in PKS 0558-504 with two lobe-like structures at $\sim 7^{\prime \prime}$ from the bright central source. The extended radio structure and the low value of the radio-loudness similar to radio-quiet Seyfert galaxies coupled with constraints from higher energy bands argue against a jet-dominated emission in PKS 0558-504. The study of the SED, which is dominated by a nearly constant optical-UV emission, supports the conclusion that PKS 0558-504 is accreting at super-Eddington rate. This conclusion was reached assuming $M_{\mathrm{BH}}=2.5 \times 10^{8} M_{\odot}$, which was obtained with a new scaling method based on X-ray spectral variability results. A comparison between the accretion luminosity and the kinetic power associated with the jet suggests that in this source the accretion power dominates in agreement with the results obtained from Radiation-MHD simulations of Galactic black holes (GBHs) accreting at the Eddington rate. The combined findings from this panchromatic investigation strongly suggest that PKS 0558-504 is a large scale analog of GBHs in their highly accreting intermediate state. Importantly, PKS 0558-504 may also be the prototype of the parent population of the very radio-loud NLS1s recently detected at $\gamma$-ray energies.

Subject headings: Galaxies: active - Galaxies: jets - Galaxies: nuclei - X-rays: galaxies
\end{abstract}

\section{INTRODUCTION}

Bipolar relativistic jets are common features in a variety of astrophysical objects, most notably in Galactic Black Holes (GBHs) and Active Galactic Nuclei (AGNs). Accretion of gas onto black holes is thought to power these collimated outflows. However, the details of the jet formation as well as the nature of the coupling between accreting matter and outflows are still among the outstanding open questions in high energy astrophysics.

Because of their vicinity and hence their high brightness, the temporal and spectral properties of GBHs are much better known and can be used to infer information on their more powerful, extragalactic analogs. Indeed considerable progress in this field has been made by multi-wavelength correlated studies of GBHs in different spectral states, which allow one to study the link between accretion (generally probed by the X-ray emission) and jet properties (in the radio regime) on "human" timescales. It is now well established that GBHs undergo state transitions, switching between two main states: the low/hard (LS) and the high/soft state (HS) passing through soft and hard intermediate states (IS), which are also named very high states (VHS) when they occur at high values of accretion rate (see McClintock \& Remillard 2006; Done et al. 2007 for recent comprehensive reviews on GBHs). Each spectral state is unambiguously characterized by a specific combination of X-ray temporal and spectral properties and by well defined radio features (see Fender et al. 2004).

In the study of the disk-jet link one of the most interesting spectral states is the IS/VHS, which is generally characterized by powerful transient relativistic ejections in the radio coupled with highly variable X-ray emission that is unambiguously associated with the accretion flow, 
unlike the LS where the origin of the X-rays is still matter of debate (e.g. Markoff et al. 2003; Zdziarski et al. 2004).

Unfortunately, the physical conditions that lead to the transient relativistic ejections during the IS/VHS are still poorly understood mostly because of their short duration in GBHs. Since the dynamical time scales are proportional to the black hole mass, in individual AGNs it is not possible to observe long-term phenomena occurring in GBHs such as the canonical spectral transitions. On the other hand, AGNs may provide better constraints on short-lived GBH phenomena and hence shed light on the jet formation and the interplay between accretion and ejection processes.

In the framework of the AGN-GBH unification, the Narrow-Line Seyfert 1 galaxies (NLS1s) are the best candidates for large-scale analogs of $\mathrm{GBH}$ s in the IS/VHS. NLS1s are historically identified by their optical emission line properties: the ratio $[\mathrm{O} \mathrm{III}] / \mathrm{H} \beta$ is less than 3 and FWHM $\mathrm{H} \beta$ is less than $2000 \mathrm{~km} \mathrm{~s}^{-1}$ (Osterbrock \& Pogge 1985; Goodrich 1989). They are seldom radio loud (Komossa et al. 2006), although recent studies reveal the existence of several NLS1s characterized by very high radio-loudness (e.g. Yuan et al. 2008). Recently, a few of these very radio-loud NLS1s have been detected at $\gamma$-ray energies by the Fermi/LAT collaboration confirming that these sources posses relativistic jets observed at small viewing angles (Abdo et al. 2009a, b. c; Foschini et al. 2009). In the X-rays, NLS1 are generally characterized by steep spectra and strong variability (e.g. Brandt et al. 1999; Leighly 1999a, b; Grupe et al. 2001). Based on these properties, it has been suggested that NLS1 are AGN in their early phase Grupe et al. 1999; Mathur 2000), characterized by relatively small black hole masses (e.g. Grupe \& Mathur 2004), and very high accretion rates in terms of Eddington units (e.g. Boroson \& Green 1992; Sulentic et al. 2000; Grupe et al. 2010; see also Marconi et al. 2008; Decarli et al. 2008 for a discording view).

PKS 0558-504 $(z=0.137)$ is the brightest radio-loud $\left(R=L_{\nu}(6 \mathrm{~cm}) / L_{\nu}(\mathrm{B}) \simeq 27\right.$, Siebert et al. 1999) NLS1 in the X-ray band and therefore one of the best studied at these energies. Previous X-ray observations with different satellites have confirmed that PKS 0558-504 shows the characteristic NLS1 properties: strong variability, steep X-ray spectrum, substantial soft excess, and relatively high luminosity. By comparing the X-ray observations from several satellites over a decade, it is evident that on long timescales the strong X-ray variability of PKS 0558-504 occurs persistently but without being accompanied by significant spectral variability: the spectrum above $2 \mathrm{keV}$ is consistently described by a powerlaw model with photon index $\Gamma \sim 2.2$ Gliozzi et al. 2000). This conclusion has been confirmed by a recent monitoring campaign with $R X T E$ : the long-term achromatic variability appears to be consistent with the behavior of GBHs during the transition LS-to-HS and inconsistent with the spectral variability typical of jet-dominated AGNs (Gliozzi et al. 2007).

In the last ten years, PKS 0558-504 has been observed repeatedly by XMM-Newton (O'Brien et al. 2001; Gliozzi et al. 2001; Brinkmann et al. 2004). The most recent and highest quality data, obtained with the $X M M-N e w t o n$ EPIC cameras from five consecutive orbits in September 2008, confirm that PKS 0558-504 is highly variable on all sampled timescales, and a spectral analysis indicates that the $2-10 \mathrm{keV}$ energy range is well fitted with a power law whose slope steepens as the source brightens and the soft excess is well described by a low-temperature Comptonization model (Papadakis et al. 2010a). These findings suggest that despite its radio loudness PKS 0558-504 appears to behave like a "normal" Seyfert galaxy from the X-ray point of view.

The main goal of this work is to shed light on the energetic of this powerful source using multi-wavelength data. For this purpose, after describing the observations and data reduction in $\S 2$, we first try to assess the role played by the jet emission beyond the radio band (in $\S 3$ ) and then to constrain the black hole mass in PKS 0558504 without the use of optical measurements (in $\S 4$ ). In Section 5 we describe the multi-wavelength behavior of PKS 0558-504 based on a 10-day multi-wavelength campaign carried out with the SWIFT XRT and UVOT simultaneously with the deep XMM-Newton observations in September 2008, and complemented with one radio observation with the Australia Telescope Compact Array (ATCA). Finally, in $\S 6$ we discuss the implications of the main results and draw our conclusions. Hereafter, we adopt a cosmology with $H_{0}=71 \mathrm{~km} \mathrm{~s}^{-1} \mathrm{Mpc}^{-1}$, $\Omega_{\Lambda}=0.73$ and $\Omega_{\mathrm{M}}=0.27$ (Bennet et al. 2003); with the assumed cosmological parameters, the luminosity distance of PKS 0558-504 is 642 Mpc.

\section{OBSERVATIONS AND DATA REDUCTION}

\subsection{SWIFT}

PKS 0558-504 was observed by the SWIFT GammaRay Burst explorer mission (Gehrels et al. 2004) between 2008 September 9 - 16. The details of this intense monitoring campaign are summarized in Tables 1 and 2 . The SWIFT X-ray Telescope (XRT; Burrows et al. 2005) observations were all performed in Windowed Timing mode (WT; Hill et al. 2004) in order to avoid the effects of pile-up. Data were reduced by the task $x r t$ pipeline version 0.12.1., which is included in the HEASOFT package 6.8. Source and background photons were selected in boxes 40 pixel long. Only single and double events (GRADES 0 to 2) were used. Source photons for the light curve and spectra were extracted with $X S$ ELECT. The auxiliary response files (ARFs) were created using xrtmkarf version 0.5.6 and the response matrix swxwt0to2s6_20010101v011.rmf.

The UV/Optical Telescope (UVOT; Roming et al. 2005) observed PKS 0558-504 in all 6 filters. The exposure times in each of the filters per segment are given in Table 1 and the corresponding magnitudes in Table 2 . The UVOT data were reduced and analyzed as described in Poole et al. (2008). Source photons were extracted from the coadded images files for each segment with a radius of 5". Backgound photons were selected in a nearby source-free region with $\mathrm{r}=20$ "! Magnitudes and fluxes were measured using the UVOT tool uvotsource. All magnitudes listed in Table 2 were corrected for Galactic reddening $\left(E_{\mathrm{B}-\mathrm{V}}=0.044\right.$; Schlegel et al. 1998). The correction factors were based on the standard reddening correction curves by Cardelli et al. (1989) as described by equation 2 in Roming et al. (2009).

2.2. XMM-Newton 
TABLE 1

SWIFT OBSERVATION LOG OF PKS 0558-504

\begin{tabular}{|c|c|c|c|c|c|c|c|c|c|c|}
\hline \multirow[t]{2}{*}{ Segment } & \multirow{2}{*}{$\begin{array}{c}\text { Start time } \\
(\mathrm{UT})\end{array}$} & \multirow{2}{*}{$\begin{array}{c}\text { End Time } \\
\text { (UT) }\end{array}$} & \multirow[t]{2}{*}{ MJD } & \multicolumn{7}{|c|}{ Observing time given in $\mathrm{s}$} \\
\hline & & & & $\mathrm{T}_{\mathrm{XRT}}$ & $\mathrm{T}_{\mathrm{V}}$ & $\mathrm{T}_{\mathrm{B}}$ & $\mathrm{T}_{\mathrm{U}}$ & TuVW1 & $\mathrm{T}_{\mathrm{UVM}} 2$ & TuvW2 \\
\hline 001 & 2008-09-07 08:10 & 2008-09-07 11:36 & 54716.41 & 2215 & 122 & 122 & 122 & 244 & 210 & 488 \\
\hline 002 & 2008-09-08 01:50 & 2008-09-08 06:50 & 54717.20 & 2429 & 199 & 199 & 199 & 399 & 358 & 798 \\
\hline 003 & 2008-09-09 09:52 & 2008-09-09 11:48 & 54718.45 & 2327 & 191 & 191 & 191 & 381 & 532 & 76 \\
\hline 004 & 2008-09-10 00:15 & 2008-09-10 18:20 & 54719.39 & 2203 & 181 & 181 & 181 & 361 & 509 & 724 \\
\hline 005 & 2008-09-11 13:21 & 2008-09-11 19:59 & 54720.69 & 1897 & 163 & 163 & 163 & 326 & 344 & 653 \\
\hline 006 & 2008-09-12 00:36 & 2008-09-12 05:37 & 54721.15 & 2008 & 155 & 155 & 155 & 310 & 483 & 621 \\
\hline 007 & 2008-09-13 15:09 & 2008-09-13 18:40 & 54722.68 & 1869 & 148 & 148 & 148 & 296 & 447 & 594 \\
\hline 008 & 2008-09-14 00:42 & $2008-09-1405: 40$ & 54723.13 & 2363 & 240 & 240 & 240 & 483 & 682 & 966 \\
\hline 009 & 2008-09-15 16:56 & 2008-09-15 21:54 & 54724.80 & 2353 & 184 & 184 & 184 & 369 & 557 & 739 \\
\hline 010 & 2008-09-16 07:20 & 2008-09-16 12:11 & 54725.90 & 2072 & 165 & 165 & 165 & 330 & 461 & 660 \\
\hline
\end{tabular}

PKS 0558-504 was observed by XMM-Newton from September 9, 2008, 1:19 UT to September 16, 2008, 12:02 UT. The EPIC pn and MOS1 cameras were operated in small window mode, the MOS2 in timing mode, and both RGS in spectroscopy mode. In the present work we only use EPIC pn data, which were processed with the XMM-Newton Science Analysis Software (SAS) 8.0. The recorded single and double events were screened to remove known hot pixels and other data flagged as bad: only data with PATTERN $\leq 4, F L A G=0$ were used. A more detailed description of the XMM-Newton data analysis can be found in Papadakis et al. (2010a).

\section{3. $A T C A$}

Radio monitoring observations of the PKS 0558-504 with the ATCA commenced in April 2006. The observation session that overlapped with the SWIFT and XMMNewton monitoring campaign was carried out on September 16, 2008, 22:50 UT. The source showed a slightly inverted spectrum between 18.5 and $4.8 \mathrm{GHz}(\alpha \simeq 0.3$, where the flux density $f_{\nu}$ is related to the spectral index $\alpha$ by the relation $\left.f_{\nu} \propto \nu^{-\alpha}\right)$. The peak flux density of $0.09 \pm 0.01 \mathrm{Jy}$ was measured at $4.8 \mathrm{GHz}$. Our previous images at this frequency indicate that almost $80 \%$ of radio intensity originates from the bright unresolved core, as shown in Figure 1. No flux density fluctuations were detected on intra-hourly timescales.

\subsection{SED fitting}

The broadband spectral analysis was performed using the XSPEC v.12.4 software package (Arnaud 1996). We used FLX2XSP in FTOOLS to transform the optical and UV fluxes into suitable units for XSPEC. All the X-ray spectra were re-binned so that each bin contained at least 20 counts for the $\chi^{2}$ statistic to be valid.

\section{RADIO PROPERTIES OF PKS 0558-504}

Despite the fact that PKS 0558-504 has been one of the first radio-loud NLS1 ever discovered, not until this project its radio properties and activity have been studied in deserving detail. Our multi-wavelength, radio monitoring and imaging program with the $A T C A$ and the VLBI shows the long-term variability pattern similar to the flat spectrum, low luminosity compact radio sources (Falcke et al. 2000). A detailed description of the

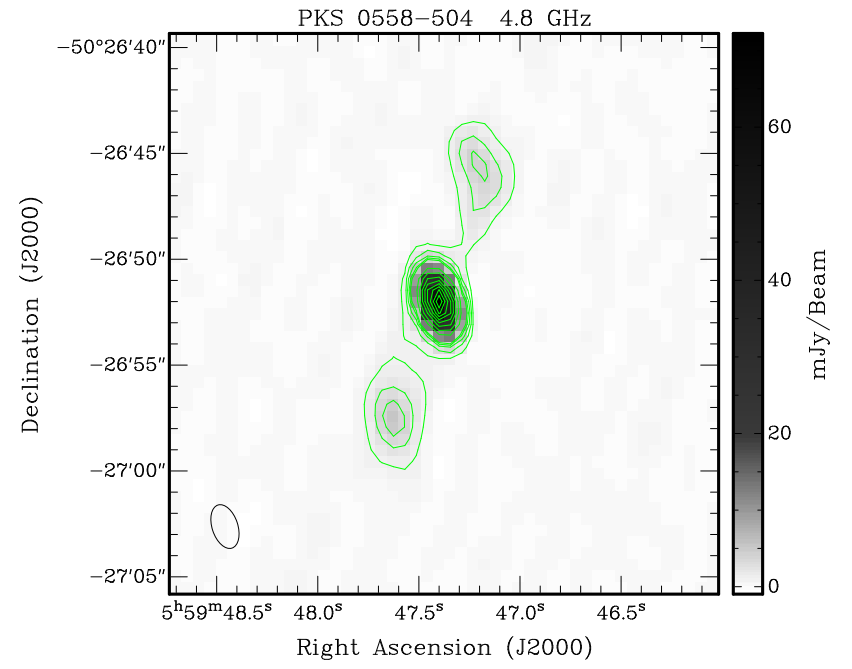

FIG. 1. - The ATCA image with the rms of $2 \times 10^{-4} \mathrm{Jy} /$ beam was obtained on the 19th of January 2008. The superimposed contour lines show isophotes between $2 \%$ and $95 \%$ of the peak flux density. The beam size is shown in the bottom left corner.

radio properties will be presented elsewhere (KedzioraChudczer et al. 2010, in preparation). Here we limit ourselves to briefly discuss the morphology of the radio emission and the intensity of the source during the $S W I F T$ and XMM-Newton campaigns in September 2008.

From Figure 1] it is evident that the radio emission of PKS 0558-504 resembles an FRI radio galaxy that is characterized by two diffuse lobes located at $\sim 7^{\prime \prime}$ from the bright, central core. At the redshift of $z=0.137$ the projected linear size of the full structure is close to $46 \mathrm{kpc}$. Interestingly, the extended radio emission observed in PKS 0558-504 is at odds with the compact radio structure generally observed in other radio-loud NLS1s, which are characterized by radio-loudness parameters larger than 100 (e.g. Yuan et al. 2008).

Historically, the radio-loudness parameter has been defined as $R \equiv L_{\nu}(6 \mathrm{~cm}) / L_{\nu}(\mathrm{B})$ with $R=10$ considered as the conventional boundary between radio-loud and radioquiet AGNs (e.g. Kellermann et al. 1994). However, Ho \& Peng (2001) demonstrated that, when the nuclear luminosities are properly measured, most of the Seyfert galaxies appear to be radio-loud, indicating that $R=10$ 
TABLE 2

SWIFT XRT COUNT RATES AND HARDNESS RATIOS AND UVOT MAGNITUdES OF PKS 0558-504

\begin{tabular}{|c|c|c|c|c|c|c|c|c|}
\hline Segment & $\mathrm{XRT}$ rate & XRT HR & $\mathrm{V}$ & B & $\mathrm{U}$ & UVW1 & UVM2 & UVW2 \\
\hline 001 & $0.747 \pm 0.020$ & $-0.11 \pm 0.02$ & $14.90 \pm 0.03$ & $15.06 \pm 0.02$ & $13.83 \pm 0.01$ & $13.54 \pm 0.01$ & $13.23 \pm 0.02$ & $13.29 \pm 0.01$ \\
\hline 002 & $1.406 \pm 0.025$ & $-0.05 \pm 0.02$ & $14.85 \pm 0.02$ & $15.07 \pm 0.01$ & $13.85 \pm 0.01$ & $13.55 \pm 0.01$ & $13.22 \pm 0.01$ & $13.30 \pm 0.01$ \\
\hline 003 & $0.933 \pm 0.021$ & $-0.12 \pm 0.02$ & $14.98 \pm 0.02$ & $15.08 \pm 0.01$ & $13.85 \pm 0.01$ & $13.63 \pm 0.01$ & $13.36 \pm 0.01$ & $13.43 \pm 0.01$ \\
\hline 004 & $0.856 \pm 0.025$ & $-0.05 \pm 0.03$ & $14.90 \pm 0.03$ & $15.07 \pm 0.02$ & $13.89 \pm 0.01$ & $13.62 \pm 0.01$ & $13.33 \pm 0.01$ & $13.33 \pm 0.01$ \\
\hline 005 & $0.808 \pm 0.022$ & $-0.15 \pm 0.03$ & $14.84 \pm 0.02$ & $15.05 \pm 0.01$ & $13.84 \pm 0.01$ & $13.57 \pm 0.01$ & $13.23 \pm 0.01$ & $13.33 \pm 0.01$ \\
\hline 006 & $1.080 \pm 0.025$ & $-0.11 \pm 0.02$ & $14.84 \pm 0.02$ & $15.05 \pm 0.01$ & $13.86 \pm 0.01$ & $13.53 \pm 0.01$ & $13.25 \pm 0.01$ & $13.30 \pm 0.02$ \\
\hline 007 & $1.010 \pm 0.025$ & $-0.07 \pm 0.02$ & $14.88 \pm 0.02$ & $15.02 \pm 0.01$ & $13.85 \pm 0.01$ & $13.54 \pm 0.01$ & $13.24 \pm 0.01$ & $13.30 \pm 0.01$ \\
\hline 008 & $0.777 \pm 0.019$ & $-0.07 \pm 0.02$ & $14.89 \pm 0.02$ & $15.02 \pm 0.01$ & $13.84 \pm 0.01$ & $13.55 \pm 0.01$ & $13.23 \pm 0.01$ & $13.31 \pm 0.01$ \\
\hline 009 & $0.577 \pm 0.017$ & $-0.13 \pm 0.03$ & $14.86 \pm 0.02$ & $15.05 \pm 0.01$ & $13.83 \pm 0.01$ & $13.56 \pm 0.01$ & $13.20 \pm 0.01$ & $13.30 \pm 0.01$ \\
\hline 010 & $0.840 \pm 0.022$ & $-0.16 \pm 0.03$ & $14.87 \pm 0.02$ & $15.04 \pm 0.01$ & $13.87 \pm 0.01$ & $13.58 \pm 0.01$ & $13.23 \pm 0.01$ & $13.31 \pm 0.01$ \\
\hline
\end{tabular}

The magnitude was corrected for reddening with $E_{\mathrm{B}-\mathrm{V}}=0.044$ given by Schlegel et al. (1998). The errors given in this table are statistical errors.

is not a reliable boundary. This conclusion is further supported by the recent findings of Sikora et al. (2007), who showed that radio-loud and radio-quiet AGNs form two distinct branches when the radio-loudness is plotted versus the Eddington ratio $\lambda_{\text {Edd }}$. Importantly, while the two branches are nearly horizontal and well separated at low values of the Eddington ratio, as $\lambda_{\text {Edd }}$ increases the branch slopes become negative and the branches broaden with substantial overlap at large values of the Eddington ratio. Unfortunately, the super-Eddington accretion rate in PKS 0558-504 (see Sect. 5.2) coupled with the lack of a clear distinction between the two branches at large values of the Eddington ratio hampers the study of the radio-loudness for PKS 0558-504 using the $\log R-\log \lambda_{\text {Edd }}$ plot.

Nevertheless, the radio-loudness of PKS 0558-504 can be quantitatively assessed by comparing it with the findings of Panessa et al. (2007) who carried out a detailed investigation of the radio-loudness in two large samples of radio-quiet Seyfert galaxies and Low-Luminosity Radio Galaxies (LLRGs). In this study both the classical radio loudness parameter $R$ and the X-ray radio loudness $R_{\mathrm{x}} \equiv \nu L_{\nu}(6 \mathrm{~cm}) / L_{2-10 \mathrm{keV}}$ were used; the latter parameter was introduced by Terashima \& Wilson (2003) to circumvent extinction problems that usually affect the optical emission and may lead to overestimates of $R$. Using nearly simultaneous radio, optical, and X-ray observations (we have used the flux values measured during the last day of the XMM-Newton - SWIFT campaign because they are almost contemporaneous with the $A T C A$ observation) for the $\log R-\log R_{\mathrm{x}}$ plot, PKS 0558-504 appears to be fully consistent with radio-quiet Seyfert galaxies and inconsistent with radio-loud objects, as visually demonstrated in Fig 2, where the boundaries have been determined by Panessa et al. (2007).

In conclusion, the extended radio emission and the symmetric location of the lobes in PKS 0558-504, which are reminiscent of the typical structure observed in FRI radio galaxies, argue against a highly beamed source. This is confirmed by the simultaneous radio-loudness parameters that are in full agreement with radio-quiet Seyfert galaxies. On the other hand, our VLBI imaging at $2.3 \mathrm{GHz}$ (Kedziora-Chudczer et al. 2010, in preparation), which shows a one-sided pc-scale jet, suggests that the presence of beamed emission cannot be ruled out for PKS 0558-504 in the radio band. For the sake of clarity

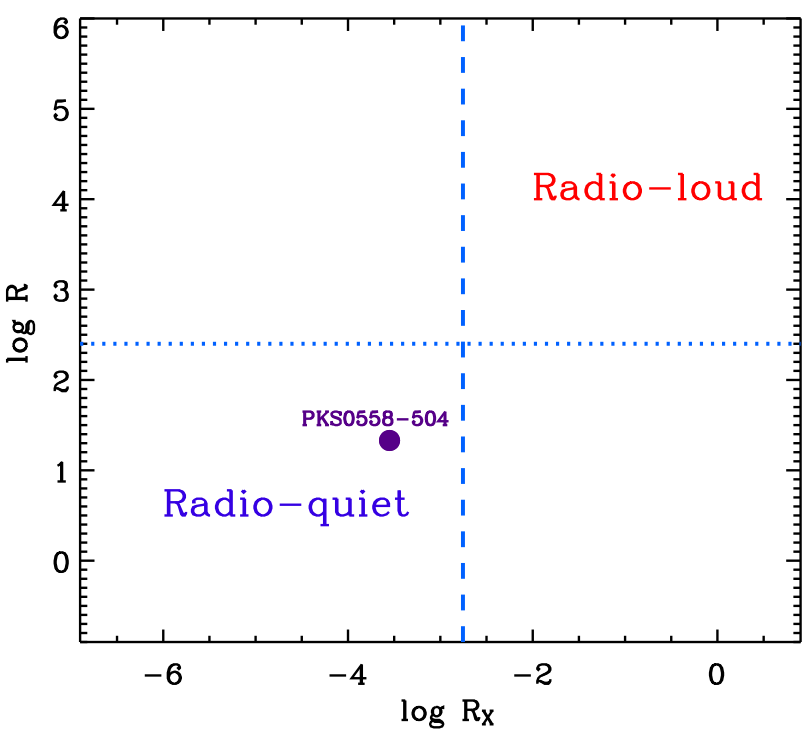

FIG. 2.- Classical radio loudness $R$ plotted versus the X-ray radio loudness $R_{\mathrm{x}}$. PKS $0558-504$ is well within the radio-quiet region and apparently inconsistent with the radio-loud objects. The boundaries between radio-loud and radio-quiet objects have been determined by Panessa et al. (2007) using two large samples of radio-quiet Seyfert galaxies and LLRGs.

and for historical reasons in the reminder of the paper we will maintain the classification of radio-loud NLS1 for PKS 0558-504.

\section{BLACK HOLE MASS OF PKS 0558-504}

In order to determine the accretion rate of PKS 0558504 and investigate its energetics, it is first necessary to constrain the mass of the supermassive black hole. Here, we report four different and independent measurements of $M_{\mathrm{BH}}$ for PKS 0558-504.

- (1) As for many AGNs for which there are no direct measurements from reverberation mapping, the $M_{\mathrm{BH}}$ in PKS 0558-504 has been estimated from the virial relationship $M_{\mathrm{BH}}=f R(\Delta V)^{2} / G$ (where $f$ is an unknown geometric factor, and $R$ and $(\Delta V)^{2}$ are the radius and velocity dispersion of broad line region, respectively). This yielded $M_{\mathrm{BH}} \simeq 6 \times 10^{7} M_{\odot}$ (Papadakis et al. 2010a), which is relatively small when compared to the typical masses of radio-loud AGNs (e.g. Mclure \& Jarvis 2004), 
but fully consistent with the values derived in NLS1s with the same method (e.g. Grupe \& Mathur 2004).

Given that the optical measurements $-L_{5100 \AA}$ (Corbin \& Smith 2000) and $H_{\beta}$ (Corbin 1997)- are non-simultaneous, and given the uncertainty on the geometric factor $f$ and the controversy about the application of the virial method to NLS1s (see e.g. Marconi et al. 2008; Decarli et al. 2008; but also Netzer 2009), it is important to constrain $M_{\mathrm{BH}}$ also with alternative techniques that are independent of optical measurements and any assumption on the BLR.

- (2) One possible alternative method is based on the so called "fundamental plane" of black holes (BHs) introduced by Merloni et al. (2003) and Falcke et al. (2004), where $M_{\mathrm{BH}}$ is related to both the X-ray and radio luminosities in any accreting $\mathrm{BH}$ systems. Recently this relationship has been refined by Gültekin et al. (2009) by utilizing only sources with $\mathrm{BH}$ masses that have been determined dynamically. Using the latter relationship in combination of quasi simultaneous measurements of the radio and X-ray emission in PKS 0558-504 we derive $M_{\mathrm{BH}} \simeq 3 \times 10^{8} M_{\odot}$, which is larger than the value obtained from the virial theorem by a factor of $\sim 4$. This finding does not depend on the nearly simultaneous nature of the observations, since we derive a very similar value for $M_{\mathrm{BH}}$ by using radio and $R X T E$ luminosities averaged over an interval of one year.

- (3) For AGNs with evenly-sampled long-term coverage in the X-ray band, a viable technique to estimate the mass of the black hole is based on the relationship $\log M_{\mathrm{BH}}=\left(\log T_{\mathrm{B}}+0.98 \log L_{\mathrm{bol}}+2.32\right) / 2.1$, where $M_{\mathrm{BH}}$ is in $10^{6} \mathrm{M}_{\odot}$ units, $L_{\text {bol }}$ is the bolometric luminosity in units of $10^{44} \mathrm{erg} \mathrm{s}^{-1}$, and $T_{\mathrm{B}}$ the time break in days derived from power spectral density (McHardy et al. 2006). PKS 0558-504 has been regularly monitored with $R X T E$ since March 2004, making it possible to estimate $M_{\mathrm{BH}}$ with the above formula. The main findings of a detailed temporal study obtained combining the long-term $R X T E$ light curve and the deep XMM-Newton observation in September 2008 are reported by Papadakis et al. (2010b) and suggest that $M_{\mathrm{BH}} \simeq 2-3 \times 10^{8} M_{\odot}$.

- (4) An additional independent way to determine the mass in $\mathrm{BH}$ systems relies on the fact that hard $\mathrm{X}$-rays are produced by the Comptonization process in both stellar and supermassive black holes. Recently, Shaposhnikov \& Titarchuk (2009) discovered that GBHs present a universal scalable relationship between the photon index and the normalization of the Bulk Motion Comptonization (BMC) model during their spectral transitions. They also demonstrate that this relationship can be used to estimate the mass of any GBHs by simply scaling the $M_{\mathrm{BH}}$ value from a suitable reference source. We have started testing the extension of this method to SMBHs and the encouraging results will be published elsewhere (Gliozzi et al. 2010 in preparation). Here we apply this method to PKS 0558-504 after describing the basic characteristics of the BMC model and of this scaling technique.

Although it was historically developed to describe the Comptonization of thermal seed photons by a relativistic converging flow (Titarchuk et al. 1997), the BMC model is a generic Comptonization model able to describe equally well the thermal Comptonization (i.e., the

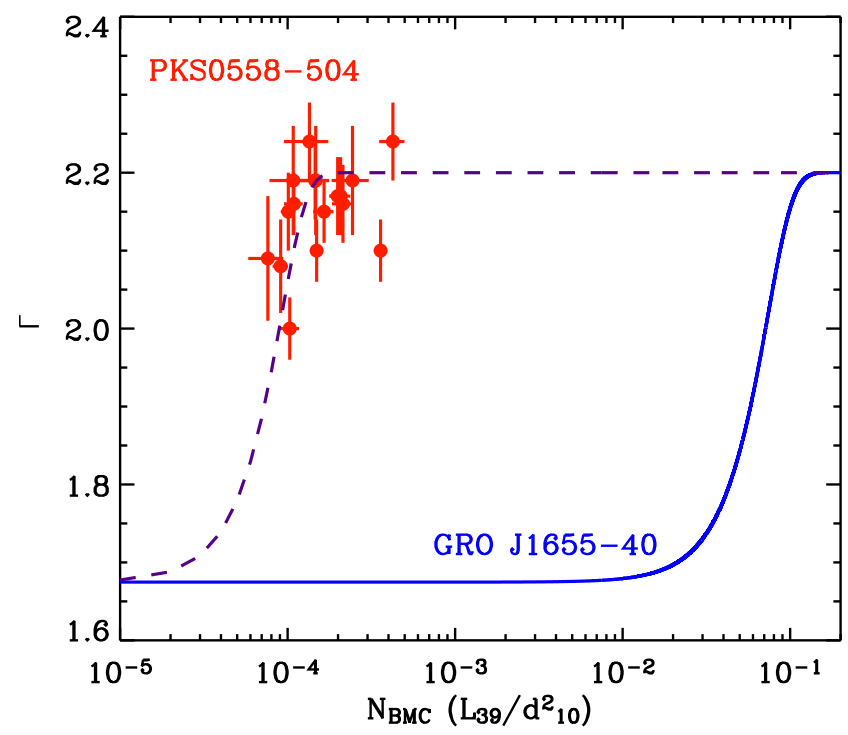

FIG. 3.- Photon index $\Gamma$ plotted versus the normalization of the Comptonization model $\mathrm{N}_{\mathrm{BMC}}$. The scaling of the black hole mass is provided by the horizontal shift between the PKS 0558-504 data points and the curve of the microquasar GRO J1655-40

inverse Compton scattering produced by electrons with a Maxwellian energy distribution) and the bulk motion Comptonization (where the seed photons are scattered off electrons with bulk relativistic motion). The BMC model is characterized by 4 free parameters: 1) the temperature of the thermal seed photons $k T, 2)$ the energy spectral index $\alpha$ (which is related to the photon index by the relation $\Gamma=1+\alpha), 3)$ a parameter $\log (A)$ related to the Comptonization fraction $f$ (.i.e., the ratio between the number of Compton scattered photons and the number of seed photons) by the relation $f=A /(1+A)$, and 4) the normalization $N_{\mathrm{BMC}}$.

In simple terms, the necessary steps to derive $M_{\mathrm{BH}}$ with this method can be summarized as follows:

1) Construct a $\Gamma-N_{\text {BMC }}$ plot for a GBH of known mass and distance, which will be used as reference (hereafter denoted by the subscript $r$ ).

2) Compute the normalization ratio between the target of interest (hereafter denoted by the subscript $t$ ) and the reference object $N_{\mathrm{BMC}, \mathrm{t}} / N_{\mathrm{BMC}, \mathrm{r}}$ at the value of $\Gamma$ measured for the AGN.

3) Derive the unknown black hole mass using the following equation

$$
M_{\mathrm{BH}, \mathrm{t}}=M_{\mathrm{BH}, \mathrm{r}} \times\left(N_{\mathrm{BMC}, \mathrm{t}} / N_{\mathrm{BMC}, \mathrm{r}}\right) \times\left(d_{t} / d_{r}\right)^{2} \times f_{G}
$$

where $M_{\mathrm{BH}, \mathrm{r}}$ is the black hole mass of the $\mathrm{GBH}$ reference object, $N_{\mathrm{BMC}, \mathrm{t}}$ and $N_{\mathrm{BMC}, \mathrm{r}}$ are the respective BMC normalizations for target and reference objects, $d_{t}$ and $d_{r}$ are the corresponding distances, and $f_{G}=\cos \theta_{r} / \cos \theta_{t}$ is a geometrical factor that depends on the respective inclination angles.

The above formula is readily obtained by considering that (a) the normalization is a function of luminosity and distance: $N_{\mathrm{BMC}} \propto L / d^{2}$, and (b) the luminosity of an accreting $\mathrm{BH}$ system can be expressed by $L \propto \eta M_{\mathrm{BH}} \dot{m}$, where $\eta$ is the radiative efficiency. The only assumptions are that different sources in the same spectral state have similar values of $\eta$ and $\dot{m}$, and that the photon index 
is a reliable indicator for the spectral state. This is in broad agreement with the positive correlation between photon index and accretion rate found in bright AGNs (e.g Shemmer et al. 2006; Papadakis et al. 2009).

Figure 3 shows the $\Gamma-N_{\mathrm{BMC}}$ diagram for PKS 0558 504 in comparison with the primary reference source GRO J1655-40, a well known microquasar whose parameters are tightly constrained: $M_{\mathrm{BH}} / M_{\odot}=6.3 \pm 0.3$, $i=70^{\circ} \pm 1^{\circ}, d=3.2 \pm 0.2 \mathrm{kpc}$ Greene et al. 2001; Hjellming \& Rupen 1995). For PKS 0558-504 we fitted the $2-10 \mathrm{keV}$ energy band of the 7 relatively long segments (with exposures up to $20 \mathrm{ks}$ ) that best describe the spectral evolution of the source during the September 2008 XMM-Newton campaign (Papadakis et al. 2010a) and the 10 short segments (2 ks exposures) simultaneous to the SWIFT observation, which will be described below. For the spectral fits, the temperature of the thermal seed photons was kept frozen at the best fit value obtained from the broadband SED fit $(k T=8-23 \mathrm{eV}$; see $\S 5.2$ ), whereas the other parameters were free to vary. The 2-10 keV spectra of all segments were adequately fitted (typically $\chi_{\text {red }} \simeq 0.9-0.95$ ) with one BMC model absorbed by Galactic $N_{\mathrm{H}}$.

As expected, due to the longer timescales associated with SMBHs, the PKS 0558-504 trend in the $\Gamma-N_{\mathrm{BMC}}$ diagram is restricted to a small portion of the trend shown by GRO J1655-40 during its 2005 spectral evolution between LS and HS. Interestingly, the short-term spectral behavior of PKS 0558-504 appears to be consistent with GRO J1655-40 in its highly accreting state.

Substituting in Eq. (1) a distance of $624 \mathrm{Mpc}$ and $N_{\mathrm{BMC}, \mathrm{t}} / N_{\mathrm{BMC}, \mathrm{r}}=8 \times 10^{-2}$ obtained from Fig. 3] we derive for PKS 0558-504 $M_{\mathrm{BH}} \simeq f_{G} 3 \times 10^{8} M_{\odot}$. Although the inclination angle of PKS $0558-504$ is unknown, the nearly symmetric position of the lobe-like radio structures suggests that the system is not seen pole-on. If we conservatively assume $i=30^{\circ}-45^{\circ}$, then $f_{G} \simeq 0.4-0.5$, leading to a mass estimate of $M_{\mathrm{BH}} \simeq 1.5 \times 10^{8} M_{\odot}$

In summary, all the optically-independent methods consistently yield $M_{\mathrm{BH}}$ values of the order of few units in $10^{8} M_{\odot}$, which is about a factor $\sim 5$ larger than the value derived from the virial theorem. Interestingly, this is in agreement with the corrective factor proposed by Marconi et al. (2008). However a systematic study of the $M_{\mathrm{BH}}$ distribution of well-defined samples of NLS1s and BLS1s obtained with optically-independent methods is necessary before drawing any general conclusion. Considering all four methods, $M_{\mathrm{BH}}$ in PKS 0558-504 ranges between $6 \times 10^{7}-3 \times 10^{8} M_{\odot}$ with a mean of $1.8 \times 10^{8} M_{\odot}$.

\section{A MULTI-WAVELENGTH VIEW OF PKS 0558-504}

\subsection{Short-term variability}

Before investigating the properties of SEDs derived from the multi-wavelength campaign in September 2008, it is important to assess the presence of variability at the different wavelengths probed by SWIFT. The light curves obtained from the six UVOT filters and from the XRT are shown in Fig. 4. The plotted optical and UV time series are the fluxes in units of $10^{-14}$ erg $\mathrm{cm}^{-2} \mathrm{~s}^{-1} \AA^{-1}$ and are corrected for Galactic absorption (see Roming et al. 2009 for details). To be conservative, we have used errorbars of $3 \sigma$ to assess the presence of significant variabil- ity with a $\chi^{2}$ test (the same conclusions are obtained with $2 \sigma$ errors), because the $1 \sigma$ statistical errors underestimate the true uncertainty on the optical-UV fluxes (see Poole et al. 2008). In the top panel of Fig. 4 in addition to the XRT light curve, we show the occurrence and duration of the XMM-Newton EPIC pn observations. Sub-intervals of the duration of $2 \mathrm{ks}$ simultaneous to the $S W I F T$ pointings were used for the SED analysis.

According to a $\chi^{2}$ test no significant variability is present in the $\mathrm{U}, \mathrm{B}, \mathrm{V}$ bands $\left(P_{\chi^{2}} \simeq 0.9-0.95\right)$ and only marginal variability is detected in the UVW1 band $\left(P_{\chi^{2}} \simeq 0.2\right)$, as suggested by a visual inspection of the four bottom panels of Fig. 4. On the other hand, the two higher energy UV bands (UVM2 and UVW2) and the $\mathrm{X}$-ray range all show statistically significant variability $\left(P_{\chi^{2}}<10^{-4}\right)$. There are however qualitative and quantitative differences between the UV and X-ray light curves: the former show only moderate variability, $F_{\text {var,M2 }}=(3.0 \pm 1.4) \%, F_{\text {var,W2 }}=(4.1 \pm 0.5) \%$ (where $F_{\text {var }}$ is the fractional variability that measures the normalized variance corrected for the statistical uncertainties), which appears to be associated with only two data points (specifically the third and fourth points in Fig. (4) that are significantly lower than the others. On the other hand, the XRT light curve shows strong variability $\left(F_{\text {var }, \mathrm{XRT}}=(28.6 \pm 0.9) \%\right)$ throughout the entire 8-day interval.

In conclusion, the SWIFT campaign in September 2008 confirms the presence of strong variability in the X-ray band of PKS 0558-504 and reveals the presence of moderate variability in the UV bands, whereas the optical bands appear to be consistent with the hypothesis of constant flux over short timescales.

\subsection{Broad-band SED}

One of the best ways to get insights into the energetic of the central engine in PKS 0558-504 and in AGNs in general is to study the broadband SED, as demonstrated by numerous successful past studies (e.g. see Elvis et al. 1994). However, one of the major problems in this field has been the lack of truly contemporaneous observations in different energy bands, which could lead to inaccurate results in virtue of the fast variability of AGNs. Recently this kind of studies has markedly improved with the advent of SWIFT thanks to its ability of observing simultaneously in several energy bands coupled with its highly flexible schedule.

Here we analyze the contemporaneous SEDs of PKS 0558-504 obtained with the SWIFT UVOT and $X M M-N e w t o n$ EPIC pn during a simultaneous campaign in September 2008. The choice of using 2000 s segments of the EPIC pn instead of the SWIFT XRT data is dictated by the much higher throughput of the EPIC camera that allows a more detailed spectral analysis of the X-ray spectrum.

Following the procedure adopted by Vasudevan \& Fabian (2009), we converted the UVOT fluxes using the FLX2XSP command in FTOOLS. The resulting spectra were then combined with the $0.3-10$ $\mathrm{keV}$ EPIC spectra and fitted in XSPEC with a model that comprises a disk and two Comptonization components: $D I S K P N+W A B S(B M C+B M C)$. Only the Comptonization models are absorbed by the Galactic $N_{\mathrm{H}}$, because the optical and UV data were already corrected for 
A panchromatic view of PKS 0558-504

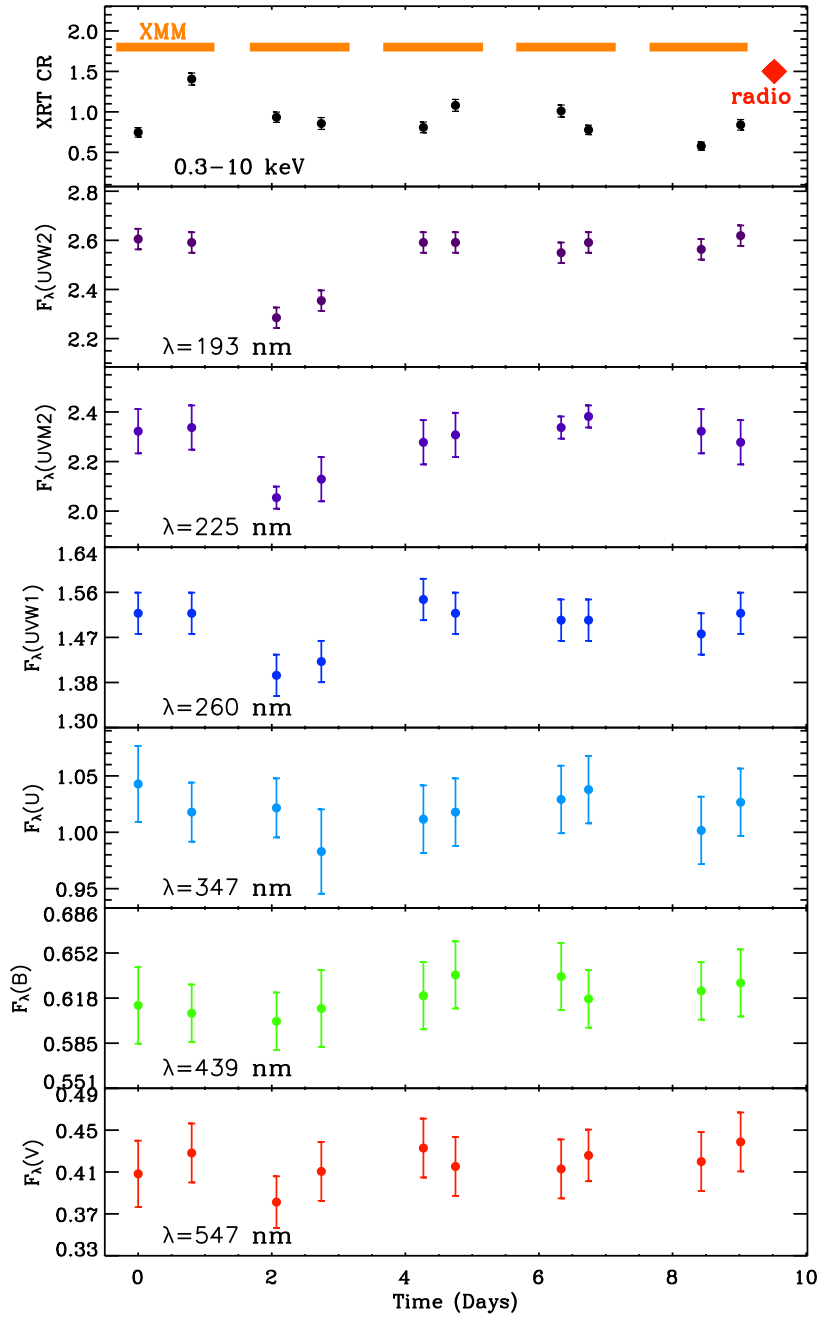

FIG. 4. - SWIFT XRT and UVOT light curves of PKS 0558-504 during the simultaneous campaign with $X M M-N e w t o n$ in September 2008. The UVOT fluxes are in units of $10^{-14} \mathrm{erg} \mathrm{cm}^{-2} \mathrm{~s}^{-1} \AA^{-1}$ and are corrected for Galactic absorption. The duration of the five $X M M$ Newton orbits is shown in the top panel as well as the occurrence of the $A T C A$ observation.

absorption.

As explained in $\S 4$ the $\mathrm{BMC}$ is a simple but comprehensive Comptonization model that can fit both thermal and bulk Comptonization processes. The use of a Comptonization model to parametrize the soft excess below 2 $\mathrm{keV}$ is guided by a thorough spectral analysis of the highest quality $X M M-N e w t o n$ spectra, which suggests that this component may arise from a hot "skin" in the innermost part of the disk in objects that accrete at rates close or above the Eddington limit (Papadakis et al. 2010a). For the hard X-rays we also used a BMC model instead of the phenomenological power law model (PL) because the BMC parameters are computed in a self-consistent way, and, unlike the PL, the power law produced by BMC does not extend to arbitrarily low energies and thus does not affect the normalization of the thermal component nor the amount of local absorption.

The DISKPN model (Gierliński et al. 1999) has three parameters: $T_{\max }, R_{\text {in }}$, and the normalization that depends on the black hole mass, the distance, the inclina- tion angle $i$, and the color factor $\beta$. The latter two quantities, which are unknown for PKS 0558-504, were kept fixed to $i=0^{\circ}$ and $\beta=1$, since they only marginally affect the luminosity derived from the direct integration of the SED (see Vasudevan \& Fabian 2009). In order to determine the maximum temperature of the accretion disk, we first fitted the UVOT data only with the DISKPN model, fixing $R_{\text {in }}=6 R_{\mathrm{G}}$ and the normalization to two different values corresponding to $M_{\mathrm{BH}}=6 \times 10^{7} \mathrm{M}_{\odot}$ and $M_{\mathrm{BH}}=2.5 \times 10^{8} \mathrm{M}_{\odot}$, respectively. The resulting best fit temperatures were respectively $\sim 23 \mathrm{eV}$ and $\sim 8 \mathrm{eV}$. If the normalization of the DISKPN model is left free to vary, the resulting value derived for the $\mathrm{BH}$ mass is $M_{\mathrm{BH}}=2.5-2.7 \times 10^{8} \mathrm{M}_{\odot}$, which in good agreement with the values inferred from the three model-independent techniques described in Sect. 4.

We then fitted the X-ray spectra by linking the temperature of seed photons of both Comptonization models to $T_{\max }$. The resulting best fit parameters for the BMC components and the corresponding luminosities are reported in Table 3 for the conservative assumption that $M_{\mathrm{BH}}=2.5 \times 10^{8} \mathrm{M}_{\odot}$. The values of the reduced $\chi^{2}$ for the X-ray spectral fits range between 0.9 and 1.2 , whereas for the overall broadband fits $\chi_{r}^{2}$ are of the order of 1.41.5. Note that the BMC parameters and the X-ray luminosity are almost insensitive of the choice of $k T$, whereas the bolometric luminosity $L_{\mathrm{bol}} \simeq L_{0.01-100 \mathrm{keV}}$ is significantly affected by this choice: $L_{\mathrm{bol}}$ increases by a factor of $\sim 3$ when $k T=23 \mathrm{eV}$ (i.e., for $M_{\mathrm{BH}}=6 \times 10^{7} \mathrm{M}_{\odot}$ ).

Since a very detailed X-ray spectral analysis has been already performed and reported elsewhere (see Papadakis et al. 2010a), here we focus on the broadband SED. Figure 5illustrates three different SEDs corresponding respectively to (a) segment 001 in the top panel, which represents the case of average optical-UV flux and average X-ray emission; (b) segment 002 in the middle panel, which refers the highest X-ray emission level; and (c) segment 003 in the bottom panel that represents the case of low optical/UV emission. Whereas the $\mathrm{X}$-ray emission varies considerably from day to day with luminosity changes up to a factor of $\sim 2$, the changes in the optical/UV band are barely noticeable and correspond to variations of $L_{\mathrm{bol}}$ lower than $10 \%$.

In conclusion, the overall SEDs of PKS 0558-504 during the September 2008 campaign are reasonably well fitted with a disk model parameterizing the optical/UV emission and two Comptonization components to describe the X-ray spectra. The SED appears to be dominated by a nearly constant disk component, with a variable X-ray contribution which represents about $1 / 100$ of the $L_{\text {bol }}$ with the assumption that $M_{\mathrm{BH}}=2.5 \times 10^{8} \mathrm{M}_{\odot}$. The X-ray contribution is further reduced by a factor of $\sim 3$ if $M_{\mathrm{BH}}=6 \times 10^{7} \mathrm{M}_{\odot}$.

\section{DISCUSSION}

\subsection{Jet contribution}

The main goal of this work is to investigate the energetic of the radio-loud NLS1 PKS 0558-504 and shed some light on the nature of its central engine. To this end, it is first necessary to assess the role played by the jet and more specifically whether the jet may dominate the high energy emission of PKS 0558-504. The findings based on the $A T C A$ observations reported in $\S 3$ - the 
TABLE 3

X-RAY SPECTRAL PARAMETERS AND LUMinOSity OF PKS 0558-504

\begin{tabular}{lccccccc}
\hline \hline Segment & $\alpha_{1}$ & $\log A_{1}$ & $\alpha_{2}$ & $\log A_{2}$ & $\begin{array}{c}L_{0.4-2 \mathrm{keV}} \\
\left(10^{44} \mathrm{erg} \mathrm{s}^{-1}\right)\end{array}$ & $\begin{array}{c}L_{2-10 \mathrm{keV}} \\
\left(10^{44} \mathrm{erg} \mathrm{s}^{-1}\right)\end{array}$ & $\begin{array}{c}L_{0.001-100 \mathrm{keV}} \\
\left(10^{46} \mathrm{erg} \mathrm{s}^{-1}\right)\end{array}$ \\
\hline 001 & $2.86_{-0.10}^{+0.07}$ & 2 & $1.09_{-0.09}^{+0.07}$ & 0.48 & 4.8 & 2.3 & 5.7 \\
002 & $2.70_{-0.08}^{+0.05}$ & 2 & $1.17_{-0.05}^{+0.04}$ & 0.64 & 10.0 & 5.3 & 5.7 \\
003 & $2.83_{-0.04}^{+0.04}$ & 2 & $1.15_{-0.05}^{+0.04}$ & 0.59 & 6.3 & 2.9 & 5.1 \\
004 & $2.70_{-0.07}^{+0.05}$ & 2 & $1.19_{-0.04}^{+0.05}$ & 0.69 & 7.5 & 3.7 & 5.1 \\
005 & $2.68_{-0.04}^{+0.04}$ & 2 & $1.10_{-0.04}^{+0.04}$ & 0.50 & 8.4 & 4.2 & 5.5 \\
006 & $2.63_{-0.07}^{+0.05}$ & 2 & $1.24_{-0.05}^{+0.05}$ & 0.86 & 10.0 & 4.6 & 5.6 \\
007 & $2.61_{-0.07}^{+0.05}$ & 2 & $1.17_{-0.05}^{+0.04}$ & 0.64 & 7.5 & 4.0 & 5.8 \\
008 & $2.72_{-0.05}^{+0.04}$ & 2 & $1.10_{-0.04}^{+0.03}$ & 0.49 & 6.1 & 3.3 & 6.0 \\
009 & $2.74_{-0.07}^{+0.07}$ & 0.7 & $1.19_{-0.06}^{+0.08}$ & 0.70 & 8.4 & 3.8 & 5.4 \\
010 & $2.81_{-0.07}^{+0.07}$ & 2 & $1.09_{-0.06}^{+0.08}$ & 0.69 & 7.4 & 3.3 & 5.8 \\
\hline \hline
\end{tabular}

Columns Table 3: $1=$ Segment of the SWIFT campaign. $2=$ Spectral index of the BMC model fitting the soft excess below 2 keV. $3=$ Measure of the Comptonization fraction $(f=a /(1+A))$ for the soft excess. $4=$ Spectral index of the BMC model for the $2-10$ keV range. $5=$ Measure of the Comptonization fraction for the $2-10 \mathrm{keV}$ range. $6=$ Soft X-ray luminosity. $7=$ Hard X-ray luminosity. $8=$ Bolometric luminosity

Notes: The bolometric luminosity is obtained by assuming $M_{\mathrm{BH}}=2.5 \times 10^{8} \mathrm{M}_{\odot}$, which leads to a best fit temperature of $k T \simeq 8$ eV. If $M_{\mathrm{BH}}=6 \times 10^{7} \mathrm{M}_{\odot}$, then $k T \simeq 23 \mathrm{eV}$ and the bolometric luminosity is a factor $\sim 3$ higher than the values reported in the Table.

radio-loudness measurements consistent with radio-quiet Seyfert galaxies and the extended and symmetric FRIlike radio structure which is at odds with the compact radio emission typical of very radio-loud NLS1s- disfavor a jet-dominated scenario, which instead appears to be the most likely scenario for the very radio-loud NLS1s recently detected with the Fermi/LAT.

This preliminary conclusion is supported by several independent pieces of evidence from studies at higher energies, which can be summarized as follows: (1) the X-ray spectral and temporal variability appears to be fully consistent with the typical behavior of radio-quiet Seyfert galaxies (Papadakis et al. 2010a, b) ; (2) the longterm spectral variability in the $2-15 \mathrm{keV}$ energy band is inconsistent with the characteristic trend observed in blazars with RXTE (Gliozzi et al. 2007); (3) unlike highly beamed AGNs, PKS 0558-504 is not detected at very high energy neither in the GeV range (the first year Fermi LAT catalog) nor at TeV energies (HESS; Giebels private communication). Combining all the information available for PKS $0558-504$ it is thus reasonable to conclude that the jet emission does not play a relevant role beyond the radio band. Therefore, for the rest of the paper we will assume that the bulk of the optical-UV-X-ray emission is associate with the accretion flow only.

\subsection{Energetic of PKS 0558-504}

The main result from the analysis of the broadband SED (described in $\$ 5$ ) is that the emission of PKS 0558-504 is dominated by a weakly variable UV bump $\left(F_{\text {var,UV }} \simeq 4 \%\right)$, which is commonly associated with the emission from the accretion disk. Conversely, the X-ray radiation, which we have interpreted as emission from two Comptonization components, appears to be highly variable $\left(F_{\text {var,x }} \simeq 30 \%\right)$ but encompasses only a small fraction of the total emission. It is instructive to assess in a more quantitative way which of the variable components (UV or X-rays) is energetically more impor- tant. Since $F_{\text {var }}$ provides a measurement of the variability normalized over the average flux, a measurement of the "true" variability (hereafter $\operatorname{var}_{\mathrm{UV}}$ and $\operatorname{var}_{\mathrm{X}}$, respectively) can be obtained by multiplying $F_{\text {var }}$ by the respective average flux. This test yields $\operatorname{var}_{\mathrm{X}} / \operatorname{var}_{\mathrm{UV}}=1.3$, indicating that the $\mathrm{X}$-ray component is the dominant variable component.

In order to get helpful insights into the nature of the central engine in PKS 0558-504 it is crucial to constrain its accretion rate in term of Eddington units. This information can be readily obtained by knowing the mass of the central black hole and measuring the bolometric luminosity. Depending on the method used (see §4) the black hole mass of PKS 0558-504 may range between $6 \times 10^{7} \mathrm{M}_{\odot}$ and $3 \times 10^{8} \mathrm{M}_{\odot}$. To be conservative, in the rest of the paper we will assume $M_{\mathrm{BH}}=$ $2.5 \times 10^{8} \mathrm{M}_{\odot}$ which corresponds to an Eddington luminosity of $L_{\mathrm{Edd}}=3.25 \times 10^{46} \mathrm{erg} \mathrm{s}^{-1}$ (note that this choice of $M_{\mathrm{BH}}$ does not affect the main findings nor our conclusions). Combining this value with the average bolometric luminosity derived from the direct integration of the broadband SED (see Table 3) leads to an accretion rate of $\lambda_{\mathrm{Edd}} \equiv L_{\mathrm{bol}} / L_{\mathrm{Edd}} \simeq 1.7$ that strongly suggests that PKS 0558-504 accretes at super-Eddington rate. Note that with the less conservative assumption of $M_{\mathrm{BH}}=6 \times 10^{7} \mathrm{M}_{\odot}$ (which corresponds to a lower Eddington luminosity and a higher bolometric luminosity), the accretion rate in Eddington units would be of the order of $\lambda_{\mathrm{Edd}} \simeq 25$.

Having measured the X-ray and bolometric luminosity over a period of ten days, it is possible to compute not only the average bolometric correction $\kappa_{2-10} \mathrm{keV} \equiv$ $L_{2-10} \mathrm{keV} / L_{\mathrm{bol}}$ but also to investigate if it varies over time. From Table 3 we infer that $\kappa_{2-10} \mathrm{keV}$ indeed varies by a factor of $\sim 2.5$ ranging between 108 and 249, with an average value of $162 \pm 39$ (where the error quoted is $1 \sigma)$. The large value of $\kappa_{2-10 \mathrm{keV}}$ appears to be in qualitative agreement with recent studies that suggest 

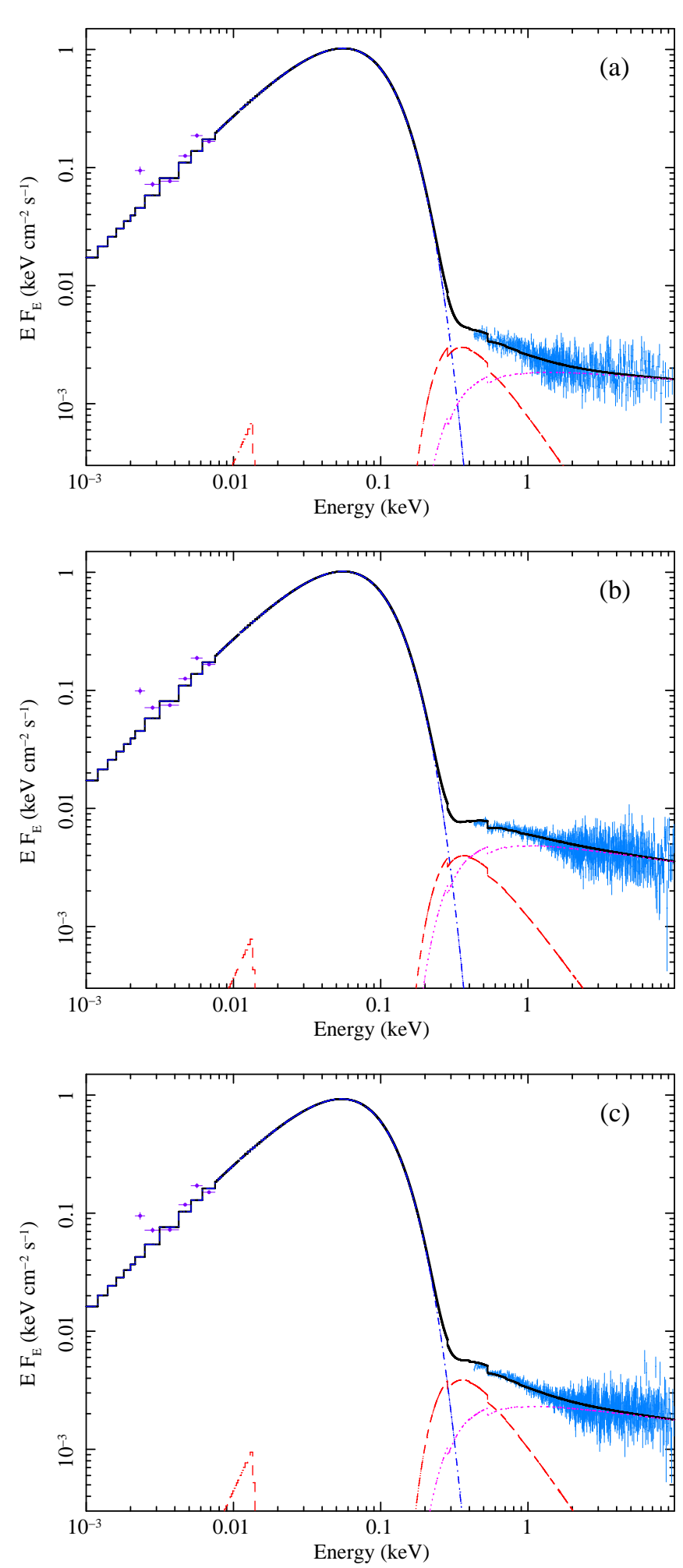

FIG. 5.- Deconvolved EPIC pn spectra in the $0.4-10 \mathrm{keV}$ energy range, combined with UVOT data. The overall SEDs are fitted with a diskpn model to parametrize the optical-UV emission and two BMC models to characterize the X-ray spectrum. The BMC models are absorbed by Galactic $N_{\mathrm{H}}$, whereas the optical/UV data have been already corrected for Galactic absorption. The spectrum in the top panel (a) represents the case of average optical-UV flux and average X-ray emission. The middle panel (b) illustrates the case of high X-ray emission, whereas the bottom panel (c) represents the case of relatively low optical/UV emission. that the X-ray bolometric correction is directly proportional to the Eddington ratio (e.g. Vasudevan \& Fabian 2007, 2009). A more quantitative comparison can be performed using the recent results presented by Lusso et al. (2010), which are based on the optical and X-ray study of a sample of 545 X-ray selected type 1 AGNs from the XMM-COSMOS survey. More specifically, plugging $\lambda_{\mathrm{Edd}}=1.7$ (the value of the Eddington ratio derived for PKS 0558-504) into equation (14) from Lusso et al. (2010) we derive $\kappa_{2-10 \mathrm{keV}}=151_{-20}^{+24}$, which is fully consistent with the average value empirically derived from the SED.

Since PKS 0558-504 possesses a radio jet, it is instructive to estimate the the inflow accretion rate and try to compare it to the the outflow mass rate associated with the jet, and similarly compare the accretion luminosity to the kinetic power. With the reasonable assumption that $L_{\mathrm{bol}}=L_{\mathrm{accr}}=\eta \dot{M}_{\mathrm{in}} c^{2}$, we derive an accretion inflow rate of $\dot{M}_{\text {in }} \simeq 1 \mathrm{M}_{\odot} /$ yr, assuming a typical radiative efficiency of $\eta=0.1$ (larger values of $\dot{M}$ are obtained if photon trapping effects play a significant role causing $\eta$ to decrease considerably). Following Merloni \& Heinz (2007) it is possible to derive an estimate of the kinetic power of the jet for radio galaxies by making use of the formula $\log L_{\text {kin }}=0.81 \log L_{\mathrm{R}}+11.9$, where $L_{\mathrm{R}}$ is the nuclear radio luminosity at $5 \mathrm{GHz}$. Using the value measured with the $A T C A, L_{5 \mathrm{GHz}}=1.7 \times 10^{41} \mathrm{erg} \mathrm{s}^{-1}$, we derive a kinetic power of the order of $L_{\text {kin }} \simeq 2 \times 10^{45} \mathrm{erg}$ $\mathrm{s}^{-1}$. The latter is considerably lower than the luminosity associated with the accretion flow, which is of the order of $5 \times 10^{46} \mathrm{erg} \mathrm{s}^{-1}$. We can estimate the outflow mass rate from $L_{\text {kin }}=(\Gamma-1) \dot{M}_{\text {out }} c^{2}$ by assuming an appropriate value for the bulk Lorentz factor. With $\Gamma=3$, which is a typical value for radio galaxies and consistent with the value inferred from the brightness temperature derived from the radio variability (Kedziora-Chudczer et al. 2010, in preparation), we derive $\dot{M}_{\text {out }} \simeq 2 \times 10^{-2} \mathrm{M}_{\odot} / \mathrm{yr}$, which is about two orders of magnitude lower than the value inferred for the accretion rate $\dot{M}_{\text {in }}$.

\subsection{Analogy with GBHs}

Our recent study based on a 1-year RXTE monitoring campaign suggests that the spectral variability behavior of PKS $0558-504$ in the $2-15 \mathrm{keV}$ energy band mimics GBHs in their highly accreting IS (Gliozzi et al. 2007). We can now test this analogy in a more quantitative way. First, we can compare the results from the energetic section relative to the disk-jet interaction with the findings of Ohsuga et al. (2009) that are derived from radiationmagneto-hydrodynamic simulations of a stellar BH system accreting at the Eddington rate. The general agreement with the simulations (specifically, $\dot{M}_{\text {out }} \ll \dot{M}_{\text {in }}$ and $L_{\text {kin }}<L_{\text {accr }}$ ) lends further support to the hypothesis that PKS 0558-504 is a large scale analog of a GBH in a highly accreting state.

The analogy with the IS/VHS is supported by the direct comparison of PKS 0558-504 with GRO J1655-40 in the $\Gamma-N_{\mathrm{BMC}}$ diagram that we used to estimate the black hole mass (see Fig. 3). Specifically, the common spectral variability trend occurs when GRO J1655-40 is switching from the LS to the HS (i.e., the source is indeed in the IS) during the rise of the outburst in March 2005 (see details in Shaposhnikov et al. 2007). Interestingly, 
the X-ray outburst of GRO J1655-40 was accompanied by enhanced emission in the radio band that faded before the peak of the X-ray outburst. Based on the values reported by Shaposhnikov et al. (2007), the ratio between radio/X-ray and radio/optical fluxes places GRO J165540 in the same lower-left quadrant of the radio-loudness plot shown in Fig. 2 for PKS 0558-504.

Further support to this conclusion comes from the comparison of the broadband spectral index $\alpha_{\mathrm{OX}}$ of PKS 0558-504 with the corresponding quantity $\alpha_{\mathrm{GBH}}$, which was recently introduced by Sobolewska et al. (2009) to characterize the broadband spectrum of GBHs and allows a direct comparison with AGNs. During our short multi-wavelength campaign in October 2008, $\alpha_{\text {OX }}$ ranged between 1.25-1.4 while the photon index varied from 2.1-2.25. Importantly, this is the very range corresponding to the intermediate state in GBHs (see Figure 6 in Sobolewska et al. 2009).

Finally, it is instructive to compare PKS 0558-504 to GRS $1915+105$, which is thought to be the only microquasar that regularly accretes at or above the Eddington limit. Interestingly, during its spectral transition hard-to-soft the X-ray spectra of GRS 1915+105 are well fitted by two BMC components (a hard component with $\Gamma_{1}=1.7-3$ and a soft component with $\Gamma_{1}=2.7$ 4.2) which evolve following the trend shown in Fig. 3 (Titarchuk \& Seifina 2009). Also for GRS 1915+105 the location of the IS/VHS in the $\Gamma-N_{\mathrm{BMC}}$ diagram is consistent with the position of PKS 0558-504 (see Figure 13 of Titarchuk \& Seifina 2009).

\subsection{Conclusion}

In conclusion, our multiwavelength analysis of the emission properties of PKS 0558-504 ranging between the radio and the hard X-rays reveals that this source has a radio jet, yet it is accreting at the Eddington level or above. All our findings appear to independently lend support to the hypothesis that PKS 0558-504 may be a large scale analog of the IS observed in GBHs. But can we extend this analogy to the whole class of NLS1s? In other words, can PKS 0558-504 be considered a prototype of NLS1s? At first sight, the "radio-loudness" of PKS 0558-504 seems to set this source in a small subclass of radio-loud NLS1. However, $R=10$ does not represent a strict boundary for the radio-loudness; indeed the radio-loudness of PKS 0558-504 appears to be fully consistent with that of radio-quiet Seyfert galaxies according to both $R$ and $R_{x}$ (see Fig. 2). Bearing in mind that PKS 0558-504 is by far the brightest NLS1 in the $\mathrm{X}$-ray band and that its X-ray luminosity is nearly 100 times the typical value of "normal" radio-quiet NLS1s, similar values of the order of $R_{x} \lesssim 10^{-3}$ for "normal" NLS1s would correspond to radio fluxes of the order of 1 mJy or less. This would explain why only a small minority of NLS1s is detected in the radio band. Additionally, it must be pointed out that in the IS the radio ejections are transients, therefore, genuine radio-quiet NLS1 might still represent large scale analogs of the IS.

It is also interesting to compare PKS 0558-504 with the radio-loud NLS1s recently detected at $\gamma$-rays. The latter are characterized by large values of the radio-loudness $(R>50)$, by compact radio emission, and by SEDs that are dominated by two broad bumps typical of jetdominated sources. Indeed, it has been argued that these very radio-loud NLS1s galaxies represent a the third class of $\gamma$-ray emitting AGNs along with blazars and radio galaxies. In this perspective, PKS 0558-504 can play a crucial role since it may represent the prototype of NLS1s with a jet seen at large viewing angles. In other words, PKS 0558-504 may be the the first representative of non-jet-dominated radio-loud NLS1s which are the parent population of the very radio-loud NLS1s detected by the Fermi/LAT, similarly to radio galaxies that are considered the non-beamed parent population of blazars.

In summary, we can hypothesize that PKS 0558-504 is indeed a prototypical NLS1. Since the jet appears to dominate only in the radio band, the emission associated with the accretion flow can be cleanly separated by that related to the jet. This makes PKS 0558-504 an ideal system to study the disk-jet interaction. In this framework, important insights into the physical conditions leading to the formation of jets in highly accreting $\mathrm{BH}$ systems can be obtained by a systematic comparison of the spectral and temporal properties of PKS 0558-504 with the corresponding properties of other highly accreting NLS1 that do not show any evidence of radio jets as well as with NLS1s that are jet-dominated. We plan to pursue this approach in our future work.

We that the anonymous referee for the constructive suggestions that have improved the clarity of the paper. MG acknowledges support by the NASA SWIFT (NNX08AU04G) and XMM-Newton (NNX08AX82G) Guest Investigator Programs and by the NASA ADP grant NNXIOAD51G. DG was supported by the NASA ADP grant (NNX07AH67G) and the NASA PSU contract (Swift is supported at Penn State by NASA contract NAS5-00136). IEP and WPB acknowledge partial support from the EU ToK grant 39965 and FP7REGPOT 206469.

\section{REFERENCES}

Abdo, A.A., et al. 2009a, ApJ, 699, 976

Abdo, A.A., et al. 2009b, ApJ, 707, 727

Abdo, A.A., et al. 2009c, ApJ, 707, L142

Arnaud, K. 1996, in ASP Conf. Ser. 101, Astronomical Data Analysis Software and Systems V, ed. G. Jacoby \& J. Barnes (San Francisco: ASP), 17

Bennet, C.L. et al. 2003, ApJS, 148, 1

Boroson, T.A. \& Green, R.F. 1992, ApJS, 80, 109

Brandt W.N., Boller Th., Fabian A.C., Ruszkowski M. 1999 MNRAS 303, L53

Brinkmann, W., Arévalo, P., Gliozzi, M., Ferrero, E. 2004, A\&A, 415, 959

Burrows, D. et al. 2005, Space Sci. Rev., 120, 165

Cardelli, J.A., Clayton, G.C., \& Mathis, J.S. 1989, ApJ, 345, 245
Corbin, M.R. 1997, ApJS, 113, 245

Corbin, M.R. \& Smith, P.S. 2000, ApJ, 532,136

Decarli, R., Dotti, M., Fontana, M., \& Haardt, F. 2008, MNRAS, 386, L15

Done, C., Gierliński, M., \& Kubota, A. 2007, A\&ARv, 15, 1

Elvis, M., et al. 1994, ApJS, 95, 1

Falcke, H., Nagar, N.M., Wilson, A.S., \& Ulvestad, J.S. 2000, ApJ, 542,197

Falcke, H., Körding, E., \& Markoff, S. 2004, A\&A, 414, 895

Fender R.P., Belloni, T.M., \& Gallo, E. 2004, MNRAS, 355, 1105 Foschini, L. et al., 2009, in Maraschi, L., Ghisellini, G., Della Ceca, R., Tavecchio, F., eds, ASP Conf. Ser., Accretion and Ejection in AGN: a Global View. Astron. Soc. Pac., San Francisco, in press (arXiv:0908.3313) 
Gehrels, N. et al. 2004, ApJ, 611, 1005

Gierliński, M., et al. 1999, MNRAS, 309, 496

Gliozzi M., Boller Th., Brinkmann W., Brandt W.N. 2000 A\&A $356, \mathrm{~L} 17$

Gliozzi M., Brinkmann, W., O’Brien, P.T., et al. 2001, A\&A 365, L128

Gliozzi M., Papadakis, I.E., \& Brinkmann W. 2007, ApJ, 656, 691

Goodrich, R.W. 1989, ApJ 342, 224

Greene, J., Bailyn, C.D., \& Orosz, J.A. 2001, ApJ, 554 1290

Grupe D., Beuermann K., Mannhein K., Thomas H.-C. 1999, A\&A 350,805

Grupe D., Thomas, H.-C, \&Beuermann, K. 2001, A\&A, 367, 470

Grupe, D. \& Mathur, S. 2004, ApJ, 606, 41

Grupe D., Komossa, S., Leighly, K.M., \& Page, K.L. 2010 ApJS, 187,64

Gültekin, K., et al. 2009, ApJ, 706, 404

Hill, J.E. et al. 2004, Proc. SPIE, 5165, 217

Hjellming, R.M. \& Rupen, M.P. 1995, Nature, 375, 464

Ho, L.C. \& Peng, C.Y. 2001,ApJ, 555, 650

Kellermann, K.I., Sramek, R.A., Schmidt, M., Green, R.F., \& Shaffer, D.B. 1994, AJ, 98, 1195

Komossa, S., Voges, W., Xu, D., et al. 2006, AJ, 132, 531

Leighly, K.M. 1999, ApJS, 125, 297

Leighly, K.M. 1999, ApJS, 125, 317

Lusso, E., et al. 2010, A\&A, 512A, 34

McClintock, J.E. \& Remillard R.A. 2006, "Compact Stellar X-ray Sources" ed. W.H.G. Lewin and M. van der Klis, Cambridge University Press, 157 (astro-ph/0306213)

[McHardy et al.(2006)]mchardy06 McHardy, I.M., Koerding, E.G., Knigge, C., Uttley, P., \& Fender, R.P. 2006, Nature, 444 730

McLure, R.J. \& Jarvis, M.J. 2004, MNRAS, 353, L45

Marconi, A., et al. 2008, ApJ, 678, 693

Markoff, S., Nowak, M., Corbel, S., Fender, R., \& Falcke, H. 2003, NewAR, 47, 491

Mathur S. 2000, MNRAS, 314, L17

Merloni, A., Heinz, S., \& di Matteo, T. 2003, MNRAS, 345, 1057

Merloni, A.\& Heinz, S. 2007, MNRAS, 381, 589
Netzer, H. 2009, ApJ, 695, 793

O'Brien, P.T., et al. 2001, A\&A, 365, L122

Ohsuga, K., Mineshige, S., Mori, M., \& Kato, Y. 2009, PASJ, 61, L70

Osterbrock, D.E. \& Pogge, R.W. 1985, ApJ, 297, 166

Panessa, F., et al. 2007, A\&A, 467, 519

Papadakis, I.E., et al. 2009, A\&A, 494, 905

Papadakis, I.E., Brinkmann, W., Gliozzi, M., et al. 2010a, A\&A, 510, A65

Papadakis, I.E., Brinkmann, W., Gliozzi, M., \& Räth, C. 2010b, A\&A, submitted

Poole, T.S. et al. 2008, MNRAS, 383, 627

Roming, P.W.A. et al. 2005, Space Sci. Rev., 120, 95

Roming, P.W.A. et al. 2009, ApJ, 690, 163

Schlegel, D. J., Finkbeiner, D. P., \& Davis, M. 1998, ApJ, 500, 525

Shaposhnikov, N., et al. 2007, ApJ, 655, 434

Shaposhnikov, N. \& Titarchuk, L. 2009, ApJ,699, 453

Shemmer, O., Brandt, W.N., Netzer, H., Maiolino, R., \& Kaspi, S. 2006, ApJ, 646

Siebert J., Leighly K.M., Laurent-Muehleisen S.A. Brinkmann W., Boller Th., Matsuoka M. 1999, A\&A 348, 678

Sikora, M., Stawarz, L., \& Lasota, J.-P. 2007, ApJ, 658, 815

Sobolewska, M.A., Gierliński, M., \& Siemiginowska, A. 2009, MNRAS, 394, 1640

Sulentic, J.W., Zwitter, T., Marziani, P., Dultzin-Hacyan, D. 2000, ApJ, 536, 5

Terashima, Y. \& Wilson, A.S. 2003, ApJ, 583, 145

Titarchuk, L.G., Mastichiadis, A. \& Kylafis, N.D. 1997, ApJ, 487, 834

Titarchuk, L.G.\& Seifina, E. 2009, ApJ, 706,1463

Vasudevan, R.V. \& Fabian, A.C. 2009, MNRAS, 381, 1235

Vasudevan, R. V. \& Fabian, A.C. 2009, MNRAS, 392, 1124

Yuan, W., et al. 2008, ApJ, 685, 801

Zdziarski, A.A., Gierliǹski, M., Miko?ajewska, J., et al. 2004, MNRAS, 351, 791 